Supporting Information

\title{
Layer-by-Layer Biomimetic Microgels for 3D Cell Culture and Nonviral Gene Delivery
}

\author{
Bruna G. Carvalho ${ }^{a}$, Franciele F. Vit ${ }^{\mathrm{a}}$, Hernandes F. Carvalho ${ }^{\mathrm{c}}$, Sang W. Han ${ }^{\mathrm{b}}$, Lucimara G. de la Torre ${ }^{\mathrm{a}}$ \\ ${ }^{a}$ Department of Material and Bioprocess Engineering, School of Chemical Engineering, University of Campinas \\ (UNICAMP), Campinas, Brazil \\ ${ }^{b}$ Department of Biophysics, Federal University of São Paulo (UNIFESP), São Paulo, Brazil \\ ${ }^{c}$ Department of Structural and Functional Biology, Institute of Biology, University of Campinas (UNICAMP), \\ Campinas, Brazil
}

* Corresponding author. Tel.: +55-19-3521-0397

E-mail address: 1torre@unicamp.br (L.G. de la Torre)

This file includes:

Figures S1 and S2

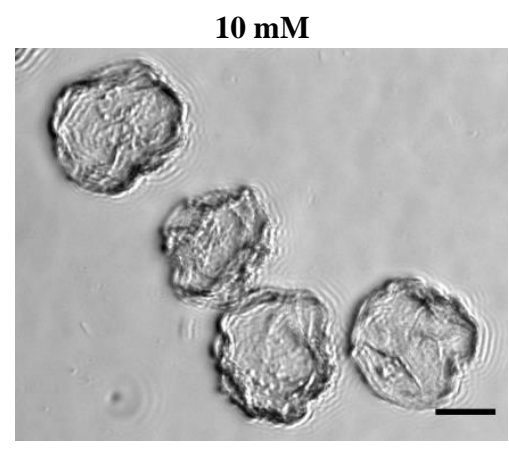

$2.5 \mathrm{mM}$

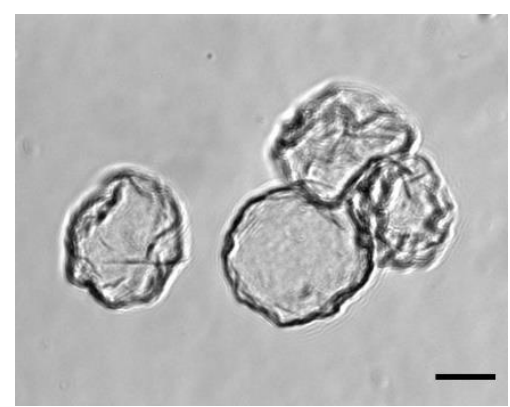

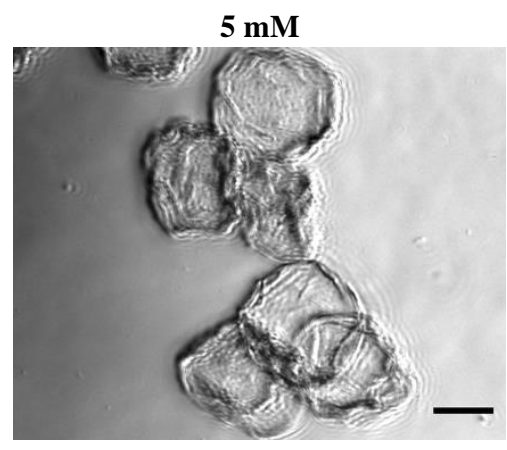

$1 \mathbf{m M}$

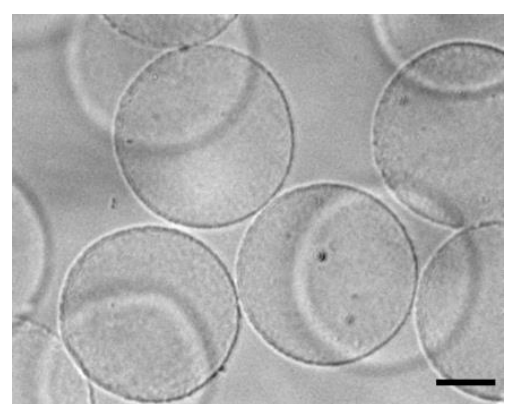

Fig. S1 - Optical microscopic images of PLL coated microgels resuspended in water. Microparticle's suspension incubated in PLL concentration $(10,5,2,5$, and $1 \mathrm{mM}$ ) incubated for $1 \mathrm{~min}$ at RT (scale bar: $25 \mu \mathrm{m}$ ). 
(A)

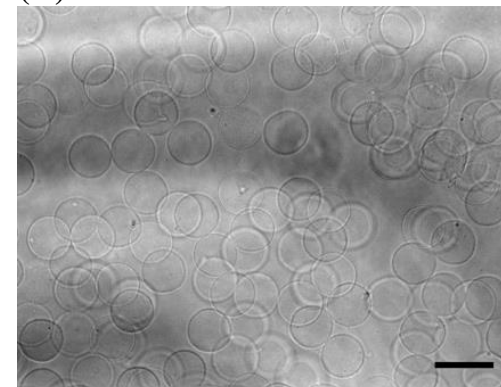

(B)

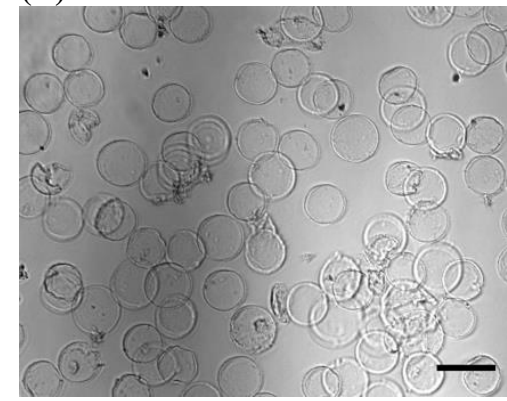

(C)

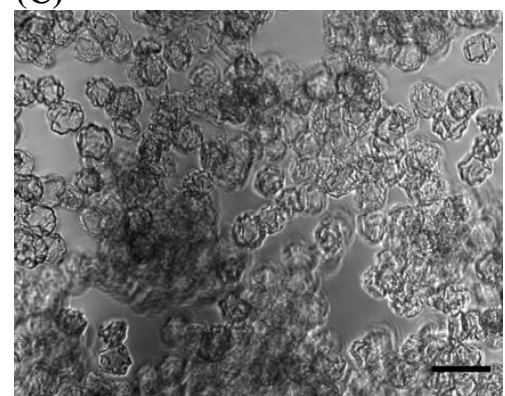

Fig. S2 - Optical microscopic images of PLL coated microgels resuspended in water. Microparticle suspension's incubated in PLL concentration (1 mM) incubated for $1 \mathrm{~min}$ at RT, (A) 1, (B) 3, and (C) 4 times. (scale bar: $100 \mu \mathrm{m}$ ). 


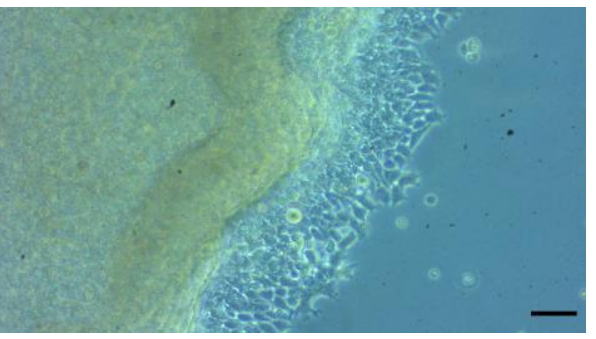

(B)

\section{Cell non-adhesive surface}

MCF-7 (without MPs)

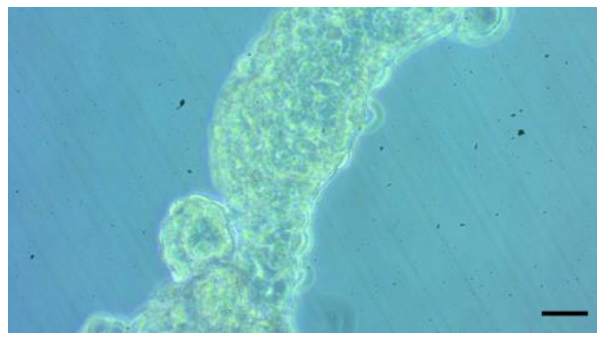

ALG/CS microgels

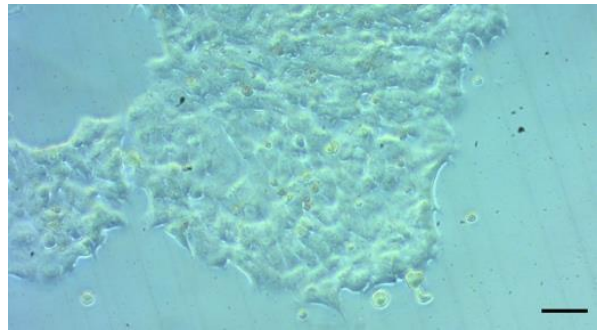

ALG microgels

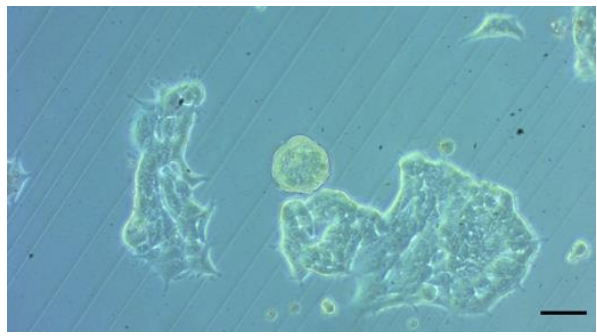

(ALG/CS) PAH/CS

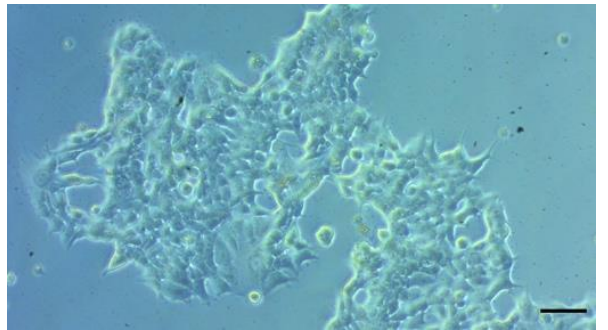

Fig. S3 - Control optical images obtained for MCF-7 cells cultured on (A) cell adhesive surface without microgel presence and (B) cell non-adhesive surface. MCF-7 cells cultured without microgel presence (I) and with microgels, ALG microgels (II) hybrid ALG-CS microgels (MP- model template particles) (III), and layered microgels (MP) PAH/Cs, CS coating (IV). Control optical images obtained for the control microgels show that cells did not attach to the microgel surface. 